\title{
Principales phases cristallines abordées dans cet ouvrage
}

Anorthite

Apatite

Aragonite

Berlinite

Beryl

Biotite

Brookite

Canasite fluorée

Carnegieite

Ca-Tschermak

Chlorapatite

Celsiane

Cordiérite

Cyclotriphosphate de sodium

Diopside

Disilicate de lithium

Enstatite

Eucryptite- $\beta$

Ferrite

Fluorapatite

Fluorine

Fluorophlogopite

Forsterite

Fresnoite

Gahnite

Gehlénite
$\mathrm{CaAl}_{2} \mathrm{Si}_{2} \mathrm{O}_{8}$

$\mathrm{Ca}_{5}\left(\mathrm{PO}_{4}\right)_{3}(\mathrm{OH}, \mathrm{Cl}, \mathrm{F})$ (formule générale, $\mathrm{A}_{5}\left(\mathrm{XO}_{4}\right)_{3} \mathrm{Z}_{q}$ ) $\mathrm{CaCO}_{3}$

$\mathrm{AlPO}_{4}$

$\mathrm{Be}_{3} \mathrm{Al}_{2} \mathrm{Si}_{6} \mathrm{O}_{18}$

$\mathrm{K}(\mathrm{Mg}, \mathrm{Fe})_{3}\left(\mathrm{AlSi}_{3}\right) \mathrm{O}_{10}(\mathrm{OH})_{2}$

$\mathrm{TiO}_{2}$

$\mathrm{Na}_{4} \mathrm{~K}_{2} \mathrm{Ca}_{5} \mathrm{Si}_{12} \mathrm{O}_{30} \mathrm{~F}_{4}$

$\mathrm{NaAlSiO}_{4}$

$\mathrm{CaAl}_{2} \mathrm{SiO}_{6}$

$\mathrm{Ca}_{5}\left(\mathrm{PO}_{4}\right)_{3} \mathrm{Cl}$

$\mathrm{BaAl}_{2} \mathrm{Si}_{2} \mathrm{O}_{8}$

$\mathrm{Mg}_{2} \mathrm{Al}_{4} \mathrm{Si}_{5} \mathrm{O}_{18}$

$\mathrm{Na}_{3} \mathrm{P}_{3} \mathrm{O}_{9}$

$\mathrm{CaMgSi}_{2} \mathrm{O}_{6}$

$\mathrm{Li}_{2} \mathrm{Si}_{2} \mathrm{O}_{5}$

$\mathrm{MgSiO}_{3}$

$\mathrm{LiAlSiO}_{4}$

$\mathrm{AFe}_{2} \mathrm{O}_{4}$

$\mathrm{Ca}_{5}\left(\mathrm{PO}_{4}\right)_{3} \mathrm{~F}$

$\mathrm{CaF}_{2}$

$\mathrm{KMg}_{3} \mathrm{AlSi}_{3} \mathrm{O}_{10} \mathrm{~F}_{2}$

$\mathrm{Mg}_{2} \mathrm{SiO}_{4}$

$\mathrm{Ba}_{2} \mathrm{TiSi}_{2} \mathrm{O}_{8}$

$\mathrm{ZnAl}_{2} \mathrm{O}_{4}$

$\mathrm{Ca}_{2} \mathrm{Al}_{2} \mathrm{SiO}_{7}$ 


\begin{tabular}{|c|c|}
\hline Hémimorphite & $\mathrm{Zn}_{4} \mathrm{Si}_{2} \mathrm{O}_{7}(\mathrm{OH}) \cdot \mathrm{H}_{2} \mathrm{O}$ \\
\hline Hydroxyapatite & $\mathrm{Ca}_{5}\left(\mathrm{PO}_{4}\right)_{3}(\mathrm{OH})$ \\
\hline Ilménite & $\mathrm{FeTiO}_{3}$ (formule générale $\mathrm{ABO}_{3}$ ) \\
\hline Kalsilite & $\mathrm{KAlSiO}_{4}$ \\
\hline KTP & $\mathrm{KTiOPO}_{4}$ \\
\hline Leucite & $\mathrm{KAlSi}_{2} \mathrm{O}_{6}$ \\
\hline LiSICON & $\mathrm{LiTi}_{2}\left(\mathrm{PO}_{4}\right)_{3}$ \\
\hline Métaphosphate de calcium & $\mathrm{Ca}\left(\mathrm{PO}_{3}\right)_{2}$ \\
\hline Métasilicate de lithium & $\mathrm{Li}_{2} \mathrm{SiO}_{3}$ \\
\hline Molybdate de sodium & $\mathrm{Na}_{2} \mathrm{MoO}_{4}$ \\
\hline Mullite & $\mathrm{Al}_{6} \mathrm{Si}_{2} \mathrm{O}_{13}$ \\
\hline NaSICON & $\mathrm{NaTi}_{2}\left(\mathrm{PO}_{4}\right)_{3}$ \\
\hline Niobate de sodium & $\mathrm{NaNbO}_{3}$ \\
\hline Niobate de lithium & $\mathrm{LiNbO}_{3}$ \\
\hline Néphéline & $\mathrm{NaAlSiO}_{4}$ ou $\mathrm{Na}_{3} \mathrm{KAl}_{4} \mathrm{Si}_{4} \mathrm{O}_{16}$ \\
\hline Norbergite & $\mathrm{Mg}_{3} \mathrm{SiO}_{4}(\mathrm{~F}, \mathrm{OH})_{2}$ \\
\hline Olivine & $(\mathrm{Mg}, \mathrm{Fe})_{2} \mathrm{SiO}_{4}$ \\
\hline Opale & $\mathrm{SiO}_{2}, \mathrm{nH}_{2} \mathrm{O}$ \\
\hline Orthophosphate de lithium & $\mathrm{Li}_{3} \mathrm{PO}_{4}$ \\
\hline Phosphate de bore & $\mathrm{BPO}_{4}$ \\
\hline Phosphate tricalcique (TCP) & $\mathrm{Ca}_{3}\left(\mathrm{PO}_{4}\right)_{2}$ \\
\hline Powellite & $\mathrm{CaMoO}_{4}$ \\
\hline Pollucite & $(\mathrm{Cs}, \mathrm{Na})_{2} \mathrm{Al}_{2} \mathrm{Si}_{4} \mathrm{O}_{12} \cdot 2 \mathrm{H}_{2} \mathrm{O}$ \\
\hline Pyrophosphate de calcium & $\mathrm{CaP}_{2} \mathrm{O}_{7}$ \\
\hline Rutile & $\mathrm{TiO}_{2}$ \\
\hline Scheelite & $\mathrm{BaMoO}_{4}$ \\
\hline Silicorhénanite & $\mathrm{Na}_{2} \mathrm{Ca}_{4}\left(\mathrm{PO}_{4}\right)_{2} \mathrm{SiO}_{4}$ \\
\hline Spinelle & $\mathrm{MgAl}_{2} \mathrm{O}_{4}$ (formule générale $\mathrm{AM}_{2} \mathrm{O}_{4}$ ) \\
\hline Spodumène- $\beta$ & $\mathrm{LiAlSi}_{2} \mathrm{O}_{6}$ \\
\hline Titanite (Sphène) & $\mathrm{CaTiSiO}_{5}$ \\
\hline Trémolite & $\mathrm{Ca}_{2} \mathrm{Mg}_{5} \mathrm{Si}_{8} \mathrm{O}_{22}(\mathrm{OH})_{2}$ \\
\hline Willemite & $\mathrm{Zn}_{2} \mathrm{SiO}_{4}$ \\
\hline Wollastonite & $\mathrm{CaSiO}_{3}$ \\
\hline YAG & $\mathrm{Y}_{3} \mathrm{Al}_{5} \mathrm{O}_{12}$ \\
\hline Yoshiokaite & $\mathrm{Ca}_{7,5} \mathrm{Al}_{15} \mathrm{SiO}_{32}$ \\
\hline Zircon & $\mathrm{ZrSiO}_{4}$ \\
\hline Zircone & $\mathrm{ZrO}_{2}$ \\
\hline Zirconolite & $\mathrm{CaZrTi}_{2} \mathrm{O}_{7}$ \\
\hline
\end{tabular}

